
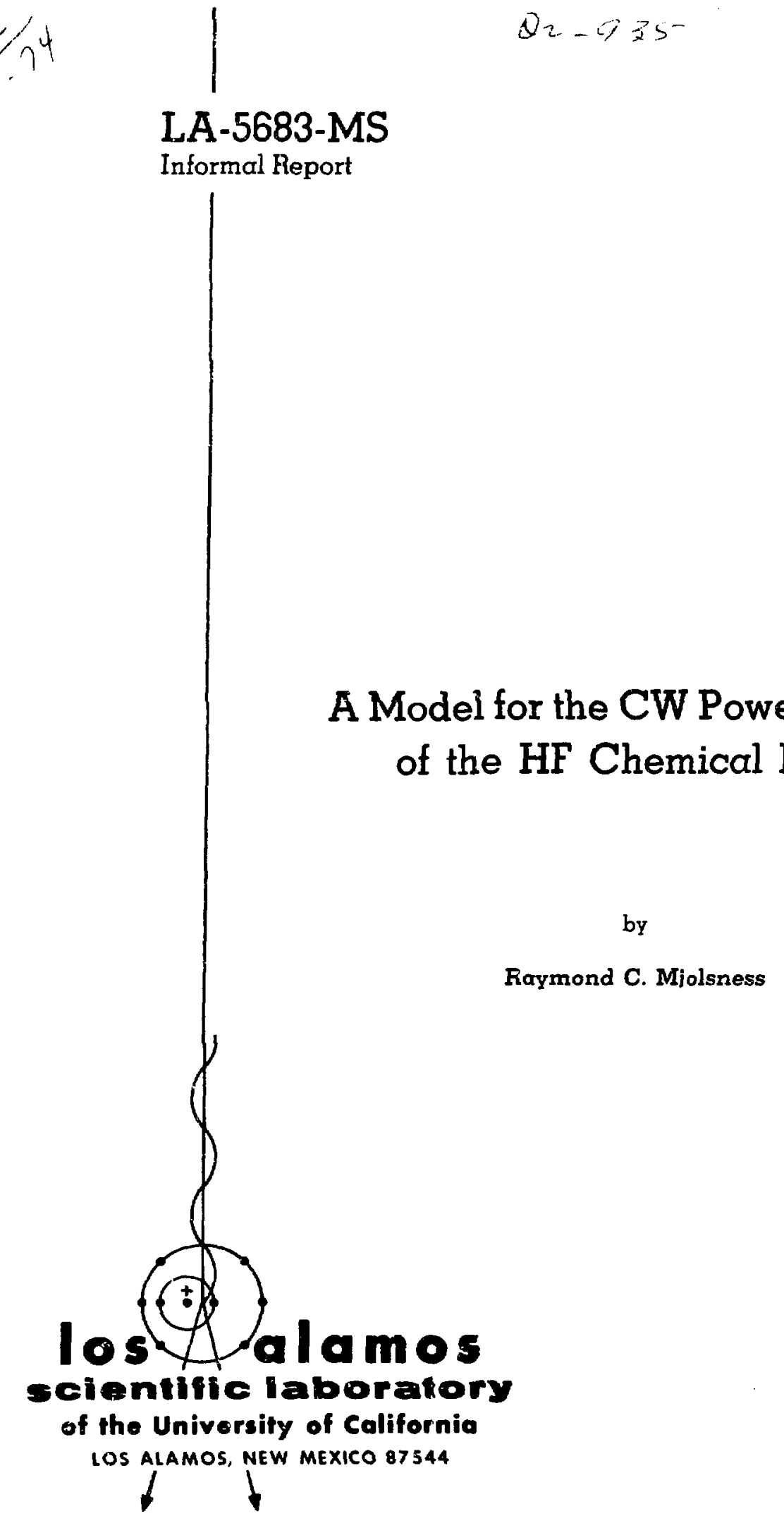

$\mathrm{UC}-34$

Reporting Date: July 1974

Issued: August 1974

\title{
A Model for the CW Power Output of the HF Chemical Laser
}

\author{
Raymond C. Miolsness
}


This report was prepared as an account of work sponsored by the United States Government. Neither the United States nor the United States Atomic Energy Commission, nor any of their employees, nor any of their contractors, subcontractors, or their employees, makes any warranty, express or implied, or assumes any legal liability or responsibility for the accuracy, completeness or usefulness of any information, apparatus, product or process disclosed, or represents that its use would not infringe privately owned rights.

In the interest of prompt distribution, this LAMS re port was not edited by the Technical Information staff.

This work was partially supported by the Air Force Weapons Laboratory under Project Order AFWL 74-032.

Printed in the United States of America. Available from

National Technical Information Service

U.S. Department of Commerce

5285 Port Royal Road

Springfield, Virginia 22151

Price: Printed Copy \$4.00 Microfiche \$1.45 


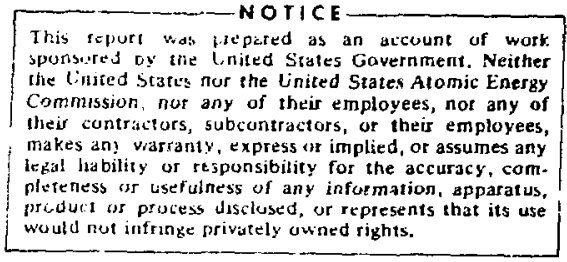

A MODEL FOS THF CW POWER OUTPUT

OF THE HF CHEMICAL LASER

by

Raymond C. Mjolsness

\begin{abstract}
A radiation model is presented for inclusion in hydrodynamics codes calculating steady-state chemical lasing conditions. The model assumes that if sufficient gain exists for lasing to occur, then P-branch lasing occurs for $3+2,2+1$ and $1+0$ transitions at the (local) $J$ value corresponding to maximum zero power galn. For simplicity, all HF states with $v>3$ are ignored. The theory ylelds the local and total power output, the local specific intensity of the radiation field and the local distribution of the HF population among the vibrational states. Very crude predictions could be obtained for the spectral distribution of the emitted radiation, although the theory is not well suited to that purpose.
\end{abstract}

\section{INTRODUCTION}

The model presented here for the $\mathrm{CW}$ chen:Ical

laser is intended for incorporation as an "optics package" for the RICE ${ }^{1}$ program, although it is not specifically constrained to that application. This work relies heavily on several studies of Emanuel $2,3,4$ and is especially indebted to his pioneering article ${ }^{2}$ clarifying the lasing phenomenology and demonstrating the applicability of a highly idealized model of the lasing process. The present model gives a slightly more complete description of the physics of the lasing process than is provided by Emanuel's two closed-form solutions. ${ }^{2,3}$ However, the principal improvement is provided by the treatment of the strong spatial nonuniformities in species concentrations and in temperature which occur $^{1}$ in the direction of laser propagation. Because of these spatial nonunfformities, the present model provides a closed-form solution only in certain approximation. Strictly, it is necessary to solve for the specific intensity of the :-itation for each $v+1+v$ transition and for the concencration of each vibrational state of HF self-consistently.
The "optics package" provides the equations for this purpose, a first approximation to a solution of the equations and a prescription for the iterative solution of the equations.

The model assumes that each volume element of the gas either does not lase or, if the zero power gain exceeds the threshold gain, lases at the ( $P-$ branch) vibrational-rotational transition with maximum zero power gain. No attempt is made to follow the evolution of the dominant lasing mades. Instead, it is simply assumed that all three transitions $3 \rightarrow 2,2 \rightarrow 1$ and $1 \rightarrow 0$ 1ase at the single $J$ value determined by the maximum zero power gain. All vibrational states with $\mathrm{y}>3$ are ignored throughout the calculation. It is assumed that all vibrationalratational lines are Doppler broadened and all gains are computed at ine center. This has the effect of assuming that for each $v+1+v$ transition lasing occurs on the single Fabry-Perot longitudinal mode closest to line center ar that, if multiple modes are excited, none is very far from line center. All hole burning effects are ignored. Some spectral iniormation is contained in the model; for at each 
poinc at which lasing occurs a single $J$ value is excited and this $\mathrm{J}$ value changes with iocation in the gas. However, this change in $J$ value is discontinuous and no provision 1 s made for the lasing on several $J$ values and the shifting of excited longitudinal modes which is typical of transition regions for $J$ shifting in actual. lasers. The modei is, of course, most sulted to describe low-pressure (<1-atm ) lasers.

The nodel adapts readily to any desired level of treatment of vibrational-transiation and vibrational-vil rational HF energy transfer reactions, since the mathematical formularton does not depend on the spectific form taken for the "chemistry" reactions. The model assumes the rigid rotator approximation for the HF energy levels, but, at the price of some algebraic complexity, this restriction couid be removed. Similarly, the restriction to $v \leqslant 3$ could be removed (and should be removed for applications in which the $\mathrm{H}+\mathrm{F}_{2} \rightarrow \mathrm{HF}+\mathrm{E}$ reaction is of importance). The matrix elements connecting the upper and lower lasing states are allowed to depend on both quantum numbers $\mathrm{J}$ and $v$. This dependence affects the populations required for threshold gain by factors of two or three. The threshold gain condition provides a set of integral constraints on the vibrational populations of $\mathrm{HF}$ in the presence of spatial inhomogenefties. It is essential to the easy applicability of the model to replace these integral constraints by algebralc constraints on the total HF vibrational populations. This replacement requires a certain approximate treatment of the temperature profile which is bastc to the model.

\section{THE FLOW CONFIGURATION}

The primary hydrodynamic flow is in the $x$ direction. Hydrogen gas and issoclated fluorine gas are admitted into the system at supersonic velocities through a series of rectangular nozzles at $x=0$. Mixing of the fluids occurs in the rdirection and hydrodynamic vartables acquire a $z$-dependence. Huwever, boutudary layer effects, which give rise to a $y$-dependence of flow quantities, w11 be ignored.

Plane parallel mirrors are assumed to be placed adjacent to the gas flow. This will be an adequate modeling of the physical situation, provided that any region existing between the gas and the mizicurs has negligible losses and provided that the laser approximates a Fabry-Perot cavity. Mirror iosses and elge effects are also 1gnored. The radiation path is thus taken to be strictly in the z-direction, and the region of the gas in which possible lasing occurs is taken to be

$x_{0}<x<x_{0}+d$,

$0<\mathrm{y}<\mathrm{B}$,

and

$0<2<\mathrm{I}$.

The configuration is depicted in Fig. 1 .

\section{RADIATIVE TRANSPORT IN THE SYSTEM}

The equations describing radiative transport have been written down many times in the extensive 11terature on the subject, but the notation and units are far from standard, even in the portion of the 1iterature relevant to $\mathrm{CW}$ lasers. Also, occastional numerical errors occur in the formulas for the constants defining the theory. Thus we will give here a consistent set of equations defining the theory in cgs units before applying the theory to

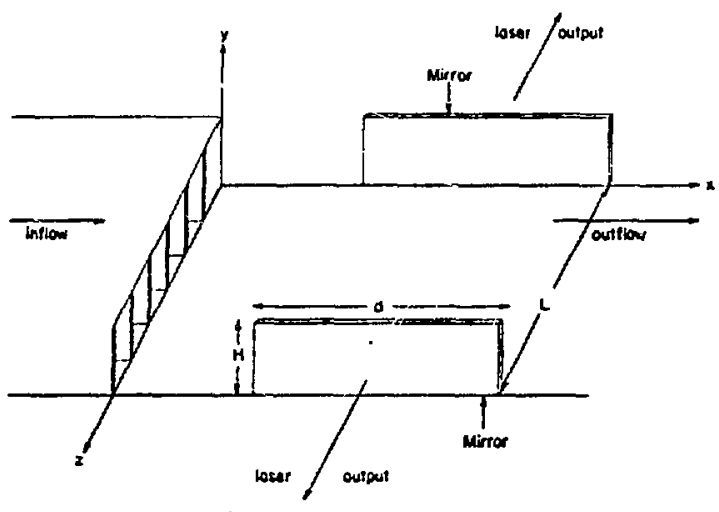

Fig. 1. The flow geometry for $\mathrm{CW}$ operation of the HF chemical laser. Basic hydrodynamic flow is in the $x$-direction with mixing in the zdirection. Radiation path is the $z$-direction. 
the calculation of laser fower sutput. We will always work in the framework of radiation transport theory. That is, no phase information is used in the evaluation of the radiation field, even though the field is coherent. The notation will follow Gilles and Vincenti ${ }^{5}$ as far as possible.

The basic quantity of the theory is the specific intensity $I_{\nu}(\vec{r}, \vec{\Omega})$ of a steady-state radiation field and is given here in units of ergs $-\mathrm{cm}^{-2}$ - sterad ${ }^{-1}$. No attempt is made to follow the time evolution of the lasing modes. The ambiguity of which of the possible lasing modes are present in the steady-state radiation field is settled by simply postulating a mode structure wilich is not unreasonable but is underived. However, the amplitudes of the permitted modes are determired selfconsistently from the transport equation and the chemical rate equations.

We denote by $B_{L U}$ the Einstein coefficient far absorptions per unit time per atom per unit solid angle. In each absorption an HF molecule is transferred from a lower lasing state $L=(v, J)$ to an upper lasing state $U=(v+1, J-1)$. We assume lasing always occurs on the $\mathrm{P}-$ branch. Then if $\mathrm{w}$ denotes the upward transition probability per unit time per steradian for an $\mathrm{HF}$ molecule in the L-state, we have

$W=B_{L U} I_{v}$.

With the above conventions the numerical value of the Einstein coefficient is given by

$\mathrm{B}_{\mathrm{LU}}=\left(\frac{8 \pi^{3}}{3 \mathrm{~h}^{2}} \mathrm{c}\right)\left(\frac{\mathrm{J}}{2 \mathrm{~J}+1}\right)\left|M_{\mathrm{v}, \mathrm{J}}^{\mathrm{v}+1, \mathrm{~J}-1}\right|^{2}$,

where $\mathrm{h}$ denotes Planck's constant, $\mathrm{c}$ the velocity of light and $M_{\mathrm{v}, J}^{\mathrm{v}+1, J-1}$ the irreducible part (independent of magnetic quantum number) of the dipole length matrix element of the transition. The above definition of $B_{L U}$ is certainly not the only one in the literature. In particular, it agrees with the conventions of Thoenes and Ratliff ${ }^{6}$ and differs by a factor of $4 \pi \times 10^{-7}$ from those of Emanuel. 4 We note in passing that the cgs units for $\mathrm{B}_{\mathrm{LU}}$ are $\mathrm{cm}^{2}-\operatorname{erg}^{-1}$ $-\sec ^{-1}$.
Numerical values are obtained from a calculation of Meredith quoted by Emanuel. ${ }^{2}$ Because the calculation is unpublished, because the calculational model is not prerise, and iecause fine details of the matrix element's value should not significantly affect the lasing population, the fit to the calculation is rather crude. A more refined fit to different data is provided by Thoenes and Ratliff. ${ }^{6}$ The features of the calculation that it was considered desirable to model were roughiy linear variations of $|M|^{2}$ with $J$ and with $v$, since these will cause factors of two or more variations in the vibrational populations. Thus the dipole length matrix element is specified by

$\left|M_{v, J}^{v+1, J-1}\right|^{2}=(1+0.06 J)(v+1) \times 10^{-38} \mathrm{erg} \mathrm{cm}^{3}$.

This formula agrees reasonably well with the Meredith calculations which are reported for $v \leqslant 2$ and with the fit of Thoenes and Ratliff. ${ }^{6}$

We will assune that the lasing lines are Doppler broadened and that the induced emission line shape $\eta_{U L}$ is equal to the absorption line shape ${ }^{*} \mathrm{LU}(v)$. The former assumption is well satisfied under usual ( 1 Torr) CW lasing conditions, but will be seriously vioiated at atmospheric pressures. The latter assumption amounts to asserting that lasing occurs on a Fabry-Perot mode sufficiently close to line center that the differing shapes ${ }^{5}$ of $\eta_{U L}$ and ${ }^{2} \mathrm{Ll}$ may be ignored. In any event, the correct Doppler broadened absorption line shape at line center $v_{0}$ satisfies

$v_{0} \div\left(v_{0}\right)=\left(\frac{M W c^{2}}{2 \pi k T}\right)^{\frac{1}{2}}$

which differs by a factor of $\sqrt{\ln 2}$ from some values $^{2,3}$ reported in the literature. In Eq. (5) M denotes the protor: mass, $W$ the molecular weight of the lasing molecule, $k$ the Boltzmann constant and $T$ the local (tra.slational) gas temperature. We assume that suffictent accuracy is provided by the approximation

$v:(v)=v_{0} p\left(v_{0}\right)$ 
We measure species concentrations in moles $/ \mathrm{cm}^{3}$ and denote the concentration of a lower (upper) lasing state by $n_{L}\left(n_{V}\right)$. These concentrations ar. related to the HF concentrations $n_{v}$ in the $v$ th $v i-$ brational state, and we approximate this relation by

$\Omega_{L}=\delta(2 J+1) e^{-\delta J(J+1)} n_{v}$,

where

$\delta=\theta_{r} / T$

and, for HF,

$\theta_{r}=30.16^{\circ} \mathrm{K} .^{2}$

Thus we assume (Justiflably) that the populations of the rotational levels are in thermodynamic equllibrium at the translational temperature $T$. We introduce the rigld rotator approximation for the energy levels as a mathematical simplification which does not alter the calculation of vibrational populations too serfously. Finally, we note that the specific. intensity of any steady state lasing mode is not significantly affected by spontaneous emisston processes and obtain as our basic transport equation for the specific intensity $I_{v}(\vec{r}, \vec{\Omega})$ for such a mode $(\Omega \cdot \nabla) I_{v}=h N_{A} v \phi(v) \quad B_{L U} \times\left[\left(\frac{2 J+1}{2 . I-1}\right) n_{U}-n_{L}\right] I_{\nu}$,

where $N_{A}$ is Avogadro's number.

We will solve Eq. (9) for the case of slab geometry in which we measure radiation propagation angle $\mu=\cos \theta$ from the 2 -axis and will assume that for any CW lasing mode we can idealtze the appropriate Fabry-Perot mode through the representation

$I_{v}=\frac{1}{2 \pi}\left(I_{v}^{+}(x, z) \delta(\mu-1)+I_{v}^{-}(x, z) \delta(\mu+1)\right)$,

where $\mathrm{I}_{v}^{+}\left(\mathrm{I}_{v}^{-}\right)$has the physical significance energy flux per unit area per unit frequency in the $+z(-2)$ direction. Because the flow quantities are assumed not to derend on the $y$-coordinates, the energy fluxes are independent of $y$ also. The further develop- ment of the lasing theory will employ certain integrals of these energy fluxes as basic variables of the theory.

\section{THRESHOLD GAIN CONDITIONS}

The gain in the possible vibrational-rotational transitions depends on the average populations in the molecular levels involved, namely

$$
\begin{aligned}
\tilde{N}_{v, J}(x, y) & =\frac{1}{L} \int_{0}^{L} d z n_{v}(x, y, z) T^{-3 / 2}(x, y, z) \\
& x \exp \left\{-J(J+1) \theta_{r} / T(x, y, z)\right]
\end{aligned}
$$

and, for the total population in the $\mathrm{s}$ th ratational level,

$$
\begin{aligned}
\tilde{N}_{T o t, J}(x, y) & =\frac{1}{L} \int_{0}^{L} d z n_{T o t}(x, y, z) T^{-3 / 2}(x, y, z) \\
& x \exp \left[-J(J+1) \theta_{I} / T(x, y, z)\right],
\end{aligned}
$$

where

$n_{\text {Tot }}=\sum_{v=0}^{3} n_{v}$.

Specifically, it follows from Eqs. (3) - (8) that the zero power gain in the $P$-branch transition $U \rightarrow L$ is given by

$\tau, \mathrm{v}, \mathrm{J}(\mathrm{x}, \mathrm{y})=\mathrm{s}_{\mathrm{o}} \boldsymbol{F}_{\mathrm{v}, \mathrm{J}}\left[\tilde{N}_{\mathrm{v}+1, \mathrm{~J}-1}-\tilde{N}_{\mathrm{v}, \mathrm{J}}\right]$,

where

$$
\begin{aligned}
S_{0} & =\left(\frac{4 \sqrt{2}}{3} \pi^{5 / 2} \times 10^{-38}\right)\left(\frac{M W}{k}\right)^{\frac{1}{2}}\left(\frac{N_{A}{ }^{O} r^{L}}{h}\right) \\
& =4.436 \times 10^{11} \mathrm{~L} \text { for } \mathrm{HF}
\end{aligned}
$$

and

$F_{\mathrm{v}, \mathrm{J}}=\mathrm{J}(1+0.06 \mathrm{~J})(\mathrm{v}+1)$. 
Thus the effective evaluation of $\tilde{N}$ becomes a centra: problem of the laser.

A first evaluation of the quantities $\tilde{N}_{v, J}$ is readily made, since it is Intended that the optics package be implemented after a hydrodynamics and chemistry (without lasing) calculation has been run and, thus, after converged values of $N_{v}(x, y, z)$ have been obtained. With these populations $\widetilde{\mathbb{N}}_{v, J}$ may be obtained and $T_{v, J}$ computed for all $v \leqslant 2$ and $J \leqslant J_{\max }$ For most purposes it should be adequate and sufficiently inexpensive of computer time to take $J_{\max }=20$.

The threshold gain $G$ is given here in terms of the mirror reflectivities $r_{0}$ at $Z=0$ and $r_{L}$ at $Z=L$ as

$G=-\ln \left(r_{0} r_{L}\right)^{\frac{1}{2}}$,

although mirror and volume losses could be included, if desired. For each computational cell in the mirror region the quantities $\tau_{\mathrm{v}, \mathrm{J}}$ are evaluated using zero-powar vibrational populations. If

$\tau, \mathrm{v}, J<G$ for $\mathrm{v} \leqslant 2$ and $\mathrm{J} \leqslant \mathrm{J}_{\max }$

then no lasing occurs in the computational cell, and no explicit changes in the hydrodynamic variables or the chemical populations need be considered. Implicit changes will occur, however, for the lasing in other portions of the gas will alter the gas temperature with effects on botn the hydrodynamics and the chemistry.

If the Inequality of Eq. (18) is violated, then lasing occurs in the coinputational ceil. It is assumed to occur oil all the permitted vibrational transitions at the P-branch rotational quantum number $J *$ defined from tho zero-power gains by the conditions

$\tau_{1, J^{\star}}=1 \leqslant J^{\operatorname{Max}} J_{\operatorname{Max}} \tau 1, J$

Then the CW lasing condition is imposed through the relations

$\mathrm{T}_{\mathrm{v}, \mathrm{J}^{*}}=\mathrm{G}, \quad$ for $0 \leqslant \mathrm{v} \leqslant 2$.
This provides a set of integral constraints on the vibrazicnal populations.

It is possible to approximate and simplify the set of integral constraints of $\mathrm{Eq} .(20)$ by noting that the difference of the exponential in the integrand of $\tilde{N}_{v, J *-1}$ and $\tilde{N}_{v, J *}$, namely

$\exp \left[-2 \mathrm{~J} * \theta_{\mathbf{r}} / T\right]$,

does not greatly affect the value and integral of Eq. (11). If $T_{0}(x)$ denotes the maximum vailie of the gas temperature profile, then the above factor may with sufficlent accuracy be replaced by

$B=\exp \left[-2 J * G_{r} / T_{0}(x)\right]$.

Such a replacement is obviously adequate when the temperature profile is flat. When it is sharply peaked the exponential factor may vary significantly with $\mathrm{z}$ but then the other exponential factor cuts off the integral much more drasticaliy. We have checked these conclusions with data from RICE calculations ${ }^{7}$ and anticipate that the approximation spcciflcied by Eq. (21) will introjuce less than a $10 \%$ error into the integral of $\mathrm{Eq}_{\mathrm{q}}$. (11) under essentially ali conditions of interest.

It is convenient to introduce the quantities

$$
\begin{aligned}
N_{v}(x, y) & =\frac{1}{L} \int_{0}^{L} d z n_{v}(x, y, z) T^{-3 / 2}(x, y, z) \\
& x \exp \left[-J^{*}\left(J^{*}-1\right) v_{r} / T(x, y, z)\right],
\end{aligned}
$$

$N_{\text {Tot }}(x, y)=\sum_{v=0}^{3} N_{v}(x, y)$

and

$N_{T o t}(x, y)=\frac{1}{2} \int_{0}^{L} i z n_{T o t}(x, y, z)$.

of course, when the flow geometry is composed of $\mathrm{N}$ equal reaction zones of width $h$, i.e. $L=N h$, only one of these zones is usually computed and the averaging operator 0 may be replaced by an average 
over $h, 1 . e$.

$0 \equiv \frac{1}{L} \int_{0}^{L} d z=\frac{1}{h} \int_{0}^{L} d z$

Normally the quantities specified by Eqs. (22) - (24) wili be assumed Independent of $y$. Then the gain conditions of Eq. (20) may be zestated as

$G=s_{0} F_{v, J *}\left|N_{v+1}-B N_{v}\right|$ for $v=0,1,2$,

a se: of equations which specify $N_{v}$ in terms of $T$, Not, G, L and $J *$.

V. INTEGRAL CONSTRAINTS ON THE VIBRATIONAL POPULATIONS

The constraints provided by Eq. (26) may be converted tu explici: solution for $N_{v}$ in terms of "Not, $B$ and the quantities

$H_{v}=\frac{G}{s_{0} F_{v, J^{*}}} \quad$ for $v=0,1,2$.

Solution of the set of linear equations for $N_{v}$ yields

$$
\begin{aligned}
N_{0}(x, y) & =\left(1-B^{4}\right)^{-1}\left[(1-B) N_{\text {Tot }}(x, y)-\left(1-B^{3}\right) H_{0}\right. \\
& \left.-\left(1-B^{2}\right) H_{1}-(1-B) H_{2}\right],
\end{aligned}
$$

$N_{1}=\left(1-B^{4}\right)^{-1}\left[B(1-B) N_{\text {Tot }}+(1-B) H_{0}\right.$

$$
\left.-B\left(1-B^{2}\right) H_{1}-B(1-B) H_{2}\right] \text {, }
$$

$N_{2}=\left(1-B^{4}\right)^{-1}\left[B^{2}(1-B) N_{\text {Tot }}+B(1-B) H_{0}\right.$

$$
+\left(1-B^{2}\right) H_{1}-B^{2}(1-B) H_{2} \mid \text {, }
$$

and

$N_{3}=\left(1-B^{4}\right)^{-1}\left[B^{3}(1-B) N_{\text {Tot }}+B^{2}(1-B) H_{O}\right.$

$$
\left.+\mathrm{B}\left(1-\mathrm{B}^{2}\right) \mathrm{H}_{1}+\left(1-\mathrm{B}^{3}\right) H_{2}\right] \text {. }
$$

Thus when values for $n_{\text {Tot }}$ and $T$ are avaflable, the integral constraints $\left(N_{v}\right)$ on the vibrational populations are provided by Eqs. (28) - (31) for any lasing region of the gas.

However, additional integrals over the vibrational populations a e required to specify the average intensity of radiation in the lasing cavity. For this purpose we suggest an approximation to the correct vibrational distribution in the gas which is consistent with Eqs. (28)-(31), correct in the limit of no $z$ variations in the system and not inconsistent with the (known) distribution of $n_{\text {Tot }}(x, y, z)$. Specifically, we define

$$
\begin{aligned}
& H_{v}(x, y, z)=\frac{1}{N} T^{3 / 2}(x, y, z) \\
& \quad \exp \left[-J^{*}\left(J^{*}-1\right) \theta_{r} / T(x, y, z)\right] H_{v}
\end{aligned}
$$

$$
\text { for } v=0,1,2
$$

and propose for the vibrational distributions

$$
\begin{aligned}
\mathrm{n}_{0}(x, y, z)= & \left(1-\mathrm{B}^{4}\right)^{-1}\left[(1-B)-\left(1-\mathrm{B}^{3}\right) \mathrm{H}_{\mathrm{o}}(\mathrm{x}, \mathrm{y}, z)\right. \\
- & \left.\left(1-\mathrm{B}^{2}\right) \mathrm{H}_{1}(\mathrm{x}, \mathrm{y}, z)-(1-\mathrm{B}) \mathrm{H}_{2}(\mathrm{x}, \mathrm{y}, \mathrm{z})\right] \\
& \mathrm{n}_{\mathrm{Tot}}(\mathrm{x}, \mathrm{y}, z)
\end{aligned}
$$

and in more compact notation,

$$
\begin{aligned}
\mathrm{n}_{1} & =\left(1-B^{4}\right)^{-1}\left[B(1-B)+(1-B) H_{0}\right. \\
& \left.-B\left(1-B^{2}\right) H_{1}-B(1-B) H_{2}\right] n_{T o t}, \\
n_{2} & =\left(1-B^{4}\right)^{-1}\left[B^{2}(1-B)+B(1-B) H_{0}\right. \\
& \left.+\left(1-B^{2}\right) H_{1}-B^{2}(1-B) H_{2}\right] n_{T O C}
\end{aligned}
$$

and 


$$
\begin{aligned}
n_{3} & =\left(1-B^{4}\right)^{-1}\left[B^{3}(1-B)+B^{2}(1-B) H_{0}\right. \\
& \left.+B\left(1-B^{2}\right) H_{1}+\left(1-B^{3}\right) H_{2}\right] n_{T O t} .
\end{aligned}
$$

We anticipate that Eqs. (33) - (36) could be used in self-consistent calculations of the flow field, the gas temperature, the radiation field and the chemical concentrations until the calculation has largely converged. Only as a last refiuement would it be necessary to turn to the rate equations for $\mathrm{n}_{\mathrm{v}}$ for an "exact" solution and, since $n_{v}$ is not used explicitly in calculations of the hydrodynamic variables and the radiation field, such "exact" solutions would sw.. a primartily to check the convergence of the calculation. The convergence properties of the theory are essentially unexamined, but it is presumably permissible to introduce exact rate equations for $n_{v}$ at any convenient stage of the iteration process.

\section{AVERAGE INTENSITY OF RADIATION}

For each of the lower vibrat lonal states $v=0$, 1,2 we define a line integrated speciflc intenstty by

$I_{v}^{ \pm}(\vec{r})=\int_{\text {emission line }} \mathrm{d} \cup \mathrm{I}_{v}^{ \pm}(\vec{r})$

The range of frequency integration is diffe:.ent in each case because of the differing vibrational constants of the lasing levels. 4 . The the gain in the system is small, an adequat . cription of the specific intensity is provided by che ave-age total density.

$I_{v}(x, y) \equiv \frac{1}{L} \int_{0}^{L} d z\left[I_{v}^{+}(\vec{r})+I_{v}^{-}\right](\vec{r})$.

In particular, the power emitted per unft area in the $x$ y plane, $P$, is determined by

$P(x, y)=G\left[I_{0}(x, y)+I_{1}(x, y)+I_{2}(x, y)\right]$

and the total power emitted is
$P=\int_{0}^{h} d y \int_{x_{0}}^{x_{0}+d} d x P \frac{e r g}{s e c}$.

The chemistry equations for the vibrational populations are uned to provide values of $I_{v}$.

VII. RADIATIVE CORRECTIONS TO HYDRODYNAMICS IND CHEMISTRY EQUATIONS

The direct effect of the volume emission of photons on the hydrodynamics of the system is a radiative correction to the time rate of change of specific total energy $E$. This correction may with sufficient accuracy be expressed in terms of the quantities $I_{v}$ and the defined quantities

$F_{v}=F_{v, J^{*}} T^{-3 / 2}(x, y, z) \exp \left[-J^{*}\left(J^{*}-1\right) \theta_{r} / T(x, y, z)\right]$

and

$g_{v}=\frac{1}{L} s_{0} F_{v}\left[n_{v+1}-B n_{v}\right]$

as

$\left[\frac{D}{D t} \rho E\right]_{\mathrm{rad}}=-\left(\mathrm{g}_{0} I_{0}+\mathrm{g}_{1} I_{1}+\mathrm{g}_{2} I_{2}\right)$

The approximate treatment of the temperature profile in Eg. (42) is consistent with the approximation introduced in the treatment of the integral constraints. The rapid variations in $z$ of the density and temperature factors justify the use of $I_{v}$. The direct effect is, of course, a decrease in temperature in the lasing region. This affects the hydrodynamic motion by changing the pressure distribution in the gas and affects the chemical reactlons in the system by changing the reaction rates.

The chemistry equations for species other than $\mathrm{HF}$ and for $\mathrm{n}_{\mathrm{TOt}}(\mathrm{x}, \mathrm{y}, \mathrm{z})$ are otherwise unchanged by the presence of lasing. The additional equations needed to complete the description of the chemical reactions may be written symbolically as

$\frac{D}{D t} n_{v}=\nabla \cdot\left(D \nabla n_{v}\right)+\left[\frac{D}{D t} n_{v}\right]_{\text {chem }}$ 


$$
+\frac{1}{N_{A} h v_{0}}\left(g_{v} I_{v}-g_{v-1} I_{v-1}\right)
$$

with the convention that

$\mathrm{I}_{-1}=0$.

The chemlcal reaction terms may be treated with any desired level of completeness, but will typically include a creation term, giving a population distribution over the vibrational states due to the reaction $\mathrm{H}_{2}+\mathrm{F}+\mathrm{HF}^{*}+\mathrm{H}$, and vibrational de-excitation terms. As an example, we display a set of reaction teras that might suffice for an approximate description of some lasing systems. The reactions and the notation are taken from a report by cohen. ${ }^{8}$ Thus

$$
\begin{aligned}
{\left[\frac{D}{D t} n_{0}\right]_{c h e m} } & =k_{4 a} n_{F} n_{H_{2}}+k_{6 a_{1}} n_{T o t} n_{1} \\
& +k_{6 b_{1}} n_{F} n_{1}+k_{6 g_{I}} n_{H_{2}} n_{1}, \\
{\left.\left[\frac{D}{D t} \cdot\right]_{1}\right]_{c h e m} } & =k_{4 b} n_{F} n_{H_{2}}+\left(k_{6 a_{2}} n_{2}-k_{6 a_{I}} n_{1}\right) n_{T o t} \\
& +\left(k_{6 b} n_{2}-k_{6 b} n_{1}\right) n_{F} \\
& +\left(k_{6 g_{2}} n_{2}-k_{6 g_{1}} n_{1}\right) n_{H_{2}},
\end{aligned}
$$

and

$$
\begin{aligned}
{\left[\frac{D}{D t} n_{2}\right]_{\text {chem }} } & =k_{4 c} n_{F} n_{H_{2}}+\left(k_{6 a_{3}} n_{3}-k_{6 a_{2}} n_{2}\right) n_{T o t} \\
& +\left(k_{6 b_{3}} n_{3}-k_{6 b_{2}} n_{2}\right) n_{F} \\
& +\left(k_{6 g_{3}} n_{3}-k_{6 g_{2}} n_{2}\right) n_{H_{2}}
\end{aligned}
$$

We emphasize, howe'er, that the theory is developed without specific limitation on the chemical reactions adopted for the system.
VIII. EVALUATION OF THE TOTAL INTENSITIES AND THE HF CONCENTRATIONS

We recall that no species diffuston occurs

through the walls at $z=0, L$. Thus a correct boundary condition at these walls is

$\frac{\partial}{\partial z} n_{v}=0 \quad$ at $z=0, L$.

A set of equations for $I_{v}$ may be obtained by averagIng Eq. (43) over the $z$-direction. Define for any quantity $Q(z)$ the average by

$$
\langle Q\rangle \equiv 00 \equiv \frac{1}{\mathrm{~L}} \int_{0}^{\mathrm{L}} d z Q(z) .
$$

Then we obtain from Eqs. (43) and (26)

$$
\begin{aligned}
\frac{G}{N_{A} L G v_{0}}\left(I_{v}-I_{v-1}\right) & =\left\langle\frac{D}{D t} a_{v}\right\rangle \\
& -\frac{\partial}{\partial x} \cdot \frac{a}{\partial x}\left\langle n_{v}\right\rangle \\
& -\left\langle\left[\frac{D}{D E} n_{v}\right]_{\text {chend }}\right\rangle .
\end{aligned}
$$

Solving for $I_{v}$ ylelds

$$
\begin{aligned}
I_{v} & =\left(\frac{N_{A} L h v_{0}}{G}\right) \sum_{j=0}^{v}\left(\left\langle\frac{D}{D t} n_{v}\right\rangle-\frac{\partial}{\partial x} p \frac{\partial}{\partial x}\left\langle n_{v}\right\rangle\right. \\
& \left.-\left\langle\left[\frac{D}{D t} n_{v}\right]_{\text {chem }}\right\rangle\right) .
\end{aligned}
$$

The quantities $I_{v}$ may be eliminated from Eq. (43) using Eq. (48). The result is a set of coupled partial integro-differential equations for the species concentrations. These equations woulf normally be solved iteratively and several iterative procedures seem natural for the problem. It is not presontly known how much flexibility there is in the choice of acceptable iterative procedure. Here we simply put forward one procedure which is consistent with the 1terative procedures adopted in the RICE ccde. 1 The procedure was propused by the RICE investigators.? 
We presume the 1 terations are started after converged values for hydrodynamic varlabjes and for species concentrations are available from runertcal evaluation of the theory without lasing. iron these variables one may complete a flrst iterative step to obtalin values $\mathrm{n}^{3}$ fior the vibrational populations and values $I^{I}$ for the total intensity by successive substitutions Into Eqs. (33) - (36) and (48). The values of $n^{1}$ obtained from Eqs. (33) - (36) should be used in Eq. (48) to obta1n $\mathbf{I}_{\mathbf{v}}^{1}$.

The iterative process may be continued by using the first iteration to provide evaluations of the radiative terms in the internal energy equations and the vibrational population equations. Specifically, we propose that the internal energy term be approximated by

$\left[\frac{D}{D t}(\rho E)^{1}\right]_{\mathrm{rad}}=-\sum \mathrm{g}_{\mathrm{v}}^{1} \mathrm{I}_{\mathrm{v}}^{?}$

where

$g_{v}^{1}=\frac{l}{L} s_{0} F_{v}\left[n_{v+1}^{1}-B n_{v}^{1}\right]$,

and the contribution to Eq. (43) be approximated by

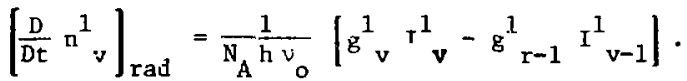

These terms would be used to correct values of the corresponding variables, as done in the other parts of the RICE program, by integrating one time step using the radiative term as the source for the time rate of change, e.g.

$\frac{\partial}{\partial t} n_{v}=\left[\frac{D}{D t} n^{1}\right]_{\mathrm{rad}}$

and, for later stages of the iterations, when $a_{v} n$

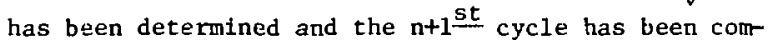
pleted through the chemical reidctions and the determination of the new radiation intensities $I_{v}{ }^{n+1}$, the radiative changes in $n_{v}$, yielding $\pi_{v}^{n+1}$ will be determined from

$$
\frac{\partial}{\partial t} \cdot n_{v}=\left[\frac{D}{D t}\left(n_{v}{ }^{n+1}\right)_{c h e m}\right]_{r a d}-\left[\frac{D}{D t} n_{v}^{n}\right]_{r a d}
$$

We presume that Eqs. (52) and (53) would be discretized by either totally explicit or totally implicit differencing to preserve the value of $n_{\text {Tot }}$ at each point. The requirement that $N_{v}$ be conserved is nore awkward to meet. The obvious way to conserve $N_{v}$, the addition of a term $\lambda n^{1}$, to the algebraic expression for $n^{2}$ with $A$ chosen to satisfy conservation, violates local conservation of $n_{\text {Tot }}$. We think that the best procedure would be to add the $n_{n}$, term for $v=0,1,2$ and then determine $n_{3}{ }^{2}$ from $n_{\text {Tot }}$. The requirement for $\mathrm{N}_{3}$ is then satisfied auts.natically; and the possible difficulty of the procedure, negative values for $n_{v}(z)$ for some range of $z$, will hopefully disappear as converged values of $n_{v}$ are reached.

Iterations would proceed by adding one link, the radlative stage, to the RICE chain of iteration

Convection - diffusion $\rightarrow$ chenistry $\rightarrow$ radiation

displayed above. In each link of the cycle the hydrodynamic variables and spectes concentrations calculated in the previous link are used to evaluate the source terms in the link under consideration. One ambiguity in this prescription is in the treatment of the vibrational populations in the radiative 11r.k. It is presentiy imagined that $u_{v}$ evaluated in the chemistry link would be used in Eq. (48) to obtain $I_{v}$. The same $n_{v}$ and $I_{v}$ would be used to evaluate the radiative source terms of Egs. (42) and (43). However, it is certainly possib le that convergence would be speeder by inserting a computation of new vibration populations $n_{v}$ from Eqs. (33) - (36) before computing new values of $I_{v}$ in the early iterative loops and omitting this step only after tho temperature distribution has largely converged. We do not presently know whtch procedure would be more efficient. 
IX. POSSIBLE MODIFICATIONS TO THE RICE CALCULAT ONAL SCHEME

We would anticipate that the discrete changes is $\partial \mathrm{n}_{\mathrm{v}} / \partial \mathrm{x}$ and $I_{\mathrm{v}}$ that occur when threshold conditions for lasing are met, when J shifting vecurs, and when cut-off occurs will cause appreciable inaccuracies in the RICE computation unless special precautions are taken to subdivide the computational cell where such a discrete change occurs. Certainly, previous ly reported calculational methods, $2,4,6$ which were essentially an integration in the $x$-coordinate only, have required the determination of the value of $x_{c}$ at which such a discontinulty occurred and the stipulation that such an $x_{c}$ be an edge of a computational cell. The location of $x_{c}$ will change during the course of the iteration, so it is necessary to determine $x_{c}$ self-consistently ouring the calculation. One procedure for accomplishing this is discussed by Emanuel. ${ }^{4}$

It is also possible that in some configurations the threshold condition for lasing is met at $x_{\text {threshold }}<x_{0}$. Under tinese conditions resulrs wi.th the RESALE program ${ }^{4}$ have been that it is necessary to modify the discontinuous change in mirror reflectivitjes at $x_{0}$ postulated in the present model in order to obtain computational results. This might be an artifact of the one-dimensional $x$-integration being used in the model. If, on the other hand, large resolved spatial variattons cause difficulties to time dependent calculations, we would exrsct the RICE program to exhibit similar phenomena. If it does, the RESAI.E program ${ }^{4}$ again provides a possible model for this feature.

\section{ACKNOWLEDGNENT}

I would like to thank Drs. T. D. Butler and W. C. Rivard and Mr. O. A. Farmer for a number of helpful discussions during the course of this work.

\section{REFERENCES}

1. W. C. Rivard, T. D. Butler, O. A. Farmer, "RICE: A Computer Program for Muiticomponent Chemically Reacting Flows at All Speeds," (LA report in preparation).

2. C. Emanuel, "Analytical Model for a Continuous Chemical Laser," J. Quant. Spectrosc. Rad1at. Transfer, 11, 1481-1520 (1971).

3. G. Emanuel and J. S. Whittier, "Closed-Form Solu tion to Rate Equations for an $\mathrm{F}+\mathrm{H}_{2}$ Laser Oscillator," The Aerospace Corporation report No. RR-0172(2756)-1, E1 Segundo, CA (1972).

4. G. Emanuel, "Resale-1: A Chemical Laser Computer Program," The Aerospace Corporation report No. TR-0172(2776)-1, E1 Segundo, CA (1972).

5. S. E. Gilles and W. G. Vincenti, "Coupled Radiative and Vibrational Nonequilibrium in a Diatom1c Gas with Applications to Gás Dynamics," J. Quant. Spectrosc. and Radiat. Transfer, 10, 71-97 (1970).

6. J. Thoenes and A. W. Ratliff, "Development and Application of Analytical Models for Chemical Lasers," Lockheed Missiles and Space Company, Inc., report No. RK-73-11, Huntsuille, AL (1973).

7. W. C. Rivard and 0. A. Farmer, Los Alamos Scicntific Laboratory, private communication.

8. N. Cohen, "A Revlew of Rate Coeffictents for Reactions In the $\mathrm{H}_{2}-\mathrm{F}_{2}$ Laser System," The Aerospace Corporation report No. TR-0172(2779)-2, E1 Segundo, CA (1971). 